\title{
Managing Stress Effectively: A Longitudinal Transtheoretical Comparison of Successful Changers, Relapsers, and Non-Changers
}

\section{N. Simay Gökbayrak ( $\nabla$ drsimay@protonmail.com )}

Cancer Prevention Research Center, University of Rhode Island

\section{Colleen Redding}

Cancer Prevention Research Center, University of Rhode Island

\section{Kerry Evers}

Pro-Change Behavior Systems, Inc.

Jessica Lipschitz

Cancer Prevention Research Center, University of Rhode Island

Miryam Yusufov

Cancer Prevention Research Center, University of Rhode Island

Andrea Paiva

Cancer Prevention Research Center, University of Rhode Island

\section{Wayne Velicer}

Cancer Prevention Research Center, University of Rhode Island Joseph Rossi

Cancer Prevention Research Center, University of Rhode Island Mark Robbins

Cancer Prevention Research Center, University of Rhode Island James Prochaska

Cancer Prevention Research Center, University of Rhode Island

\section{Research Article}

Keywords: stress management, transtheoretical model, stage of change, computer-tailored intervention

Posted Date: March 1st, 2021

DOI: https://doi.org/10.21203/rs.3.rs-208961/v1

License: (1) (1) This work is licensed under a Creative Commons Attribution 4.0 International License. Read Full License 


\section{Abstract}

Background. Stress levels among Americans are considerable. This research examined Transtheoretical Model of Behavior Change (TTM) constructs for stress management in groups organized by longitudinal progress (dynatypes): Maintainers, Relapsers, and Stable Non-Changers.

Methods. Secondary data analysis of a computer-tailored intervention group examined construct use over time across the three groups. Adults $(n=427)$ meeting criteria for not engaging in stress management behaviors at baseline comprised the analytic sample. Participants received three TTM-tailored feedback interventions to help facilitate change at baseline, 3 and 6 months. Demographics, Stage of Change, 10 Processes of Change, Decisional Balance (Pros and Cons), and Self-Efficacy were assessed at baseline, 6 , 12, and 18 months. Repeated measures MANOVA followed by ANOVAs, with Tukey follow-up tests assessed differences in use of TTM constructs longitudinally across dynatype groups.

Results. Ten of the 13 TTM constructs differentiated between Successful Changers and Stable NonChangers at baseline and over time. Relapsers were more similar to Successful Changers than to Stable Non-Changers in their use of all constructs, except Self-Efficacy.

Conclusion. Findings suggest that baseline cognitive and behavioral constructs can improve prediction of different intervention outcomes 18 months later.

\section{Introduction}

Polls indicate that stress levels among the US population remain considerable (American Psychological Association, 2017a; 2017b; 2018; 2019; Gallup, 2019). An international poll found that more than half of Americans reported experiencing stress which was one of the highest rates surpassing the global average at $35 \%$, placing the US at number seven out of 143 countries studied (Gallup, 2019). Furthermore, in the context of the unfolding COVID-19 pandemic, $45 \%$ of Americans reported that "worry and stress" related to COVID-19 "has had a negative impact on their mental health," a significant increase from $32 \%$ reported only one month earlier (Kaiser Family Foundation, 2020).

Stress is a process of negative appraisal of events and one's ability to cope with events (Lazarus \& Folkman, 1984). Unmanaged daily stress can turn into chronic stress. Chronic stress has shown to negatively impact multiple organ systems leading to a range of preventable acute and chronic illnesses (Baum \& Posluszny, 1999) as well as accelerated aging (Wikgren et al., 2012). A significant portion of Americans who suffer from chronic stress are not sufficiently practicing effective stress management behaviors essential to reduction and prevention of chronic stress (APA, 2017a). Stress management is the practice of behaviors, such as engaging in regular physical activity and seeking and maintaining healthy social support, allowing one to cope effectively with cognitive and physical symptoms associated with stress (Murphy, 1996). 
Given the prevalence of high stress levels in the US and health implications of poor stress management, wide-reaching, scalable interventions for stress management are imperative. Online interventions have advantages such as being efficient and effective for disseminating population-based research targeting public health issues including stress (Hester \& Miller, 2006).

One meta-analysis of occupational stress management interventions $(n=36)$ included workplace grouptraining, individual counseling, self-taught techniques, or a combination of diverse methods (Richardson \& Rothstein, 2008), although no methods were disseminated online. Although the medium to large effect sizes indicated good effectiveness, the average intervention duration ( 7.4 weeks with a range of 3 days to 28 weeks) may be prohibitive for many people. In addition, many people lack access to workplace interventions.

A more recent meta-analysis of web- and computer-based stress management interventions $(n=23)$ included a range of intervention durations between 2 and 12 weeks (Heber et al., 2017) and various treatment types including cognitive behavioral therapy, third-wave therapies, and alternative interventions. Stress results from 1-3-month follow-ups showed a small effect size and, for 4-6-month follow-ups, a medium effect size (Heber et al., 2017). Longer term maintenance or relapse after 6 months was not assessed.

Accordingly, one randomized trial recruited a large group of participants who were not practicing stress management. The Transtheoretical Model (TTM)-tailored computerized stress management feedback was delivered at three-time points during the first 6-months and assessed every six months at follow-up until 18 months (Evers et al., 2006). Approximately $60 \%$ of treatment group participants began practicing effective stress management skills by the 6-month time point and maintained this behavior over 18 months, compared to $37 \%$ of the assessment-only control group (Evers et al., 2006). This intervention demonstrated significant positive effects on stress, depression, and stress management behaviors (Evers et al., 2006). These intervention effects were partially replicated in a separate multiple behavior trial (Prochaska et al., 2008).

TTM is a model of intentional behavior change designed to reach individuals at all levels of readiness to change specific behaviors. As such, it is widely used to develop, guide, and evaluate health behavior interventions, including smoking cessation (Prochaska et al., 2001; Prochaska et al., 2005; Prochaska et al., 2004), exercise (Blaney et al., 2012; Johnson et al., 2008; Prochaska et al., 2008), healthy eating (Johnson et al., 2008), depression prevention (Levesque et al., 2011) and stress management (Evers et al., 2006; Prochaska et al., 2008). TTM-tailored interventions provide feedback based on individuals' Stage of Change, or their readiness to change a target behavior, and three additional TTM constructs reflecting their efforts to change (Blissmer et al., 2010; Redding et al., 2011). Decisional Balance refers to individuals' perceptions of the importance of Pros (advantages) compared to Cons (disadvantages) of behavior change (Hall \& Rossi, 2008; Prochaska et al., 1994). Self-Efficacy refers to the confidence level one feels in one's ability to successfully change a behavior across a variety of challenging situations (Velicer et al.,1990). Processes of Change reflect the frequency with which an individual engages in ten 
experiential (cognitive/affective strategies) and behavioral activities or strategies shown to facilitate forward movement through the stages (Evers et al., 2006) (Table 1 defines TTM constructs and shows sample items).

Table 1. Definitions and examples of stress management assessment items for decisional balance (pros and cons), self-efficacy and processes of change (experiential and behavioral) 


Construct Definition Assessment Item Example

Decisional Balance

Pro A positive of engaging in effective stress I would feel better about myself

if I

management managed stress in a healthy way.

Con A drawback of engaging in effective stress It is easier to use unhealthy

strategies to

management

manage my stress.

\section{Self-Efficacy}

Level of confidence in one's own ability to successfully

I am frustrated.

change a behavior across challenging situations

Experiential Processes of Change

\begin{tabular}{|c|c|c|}
\hline $\begin{array}{l}\text { Consciousness } \\
\text { Raising }\end{array}$ & $\begin{array}{l}\text { Increasing information about } \\
\text { self and problem }\end{array}$ & $\begin{array}{l}\text { I search for information about how to } \\
\text { with stress in a healthy way. }\end{array}$ \\
\hline Dramatic Relief & $\begin{array}{l}\text { Experiencing and expressing } \\
\text { feelings about one's problems } \\
\text { and solutions }\end{array}$ & $\begin{array}{l}\text { I get upset when people ignore health } \\
\text { warnings of not practicing stress } \\
\text { management. }\end{array}$ \\
\hline
\end{tabular}

Environmental Assessing how one's problem

Reevaluation affects physical environment

I think about how managing my stress in a healthy way would have a positive effect on the people around me.

Self-reevaluation Assessing how one feels and things about oneself with respect to a problem

Social liberation Increasing alternatives for non-problem behaviors available in society

I see myself as a more responsible person when I manage my stress.

I notice stress management techniques being discussed more openly.

Behavioral Processes of Change

Counterconditioning Substituting alternatives for problem behaviors

Stimulus Control

Helping

relationships

Reinforcement management
Avoiding or countering stimuli that elicit problem behaviors

Being open and trusting about problems with someone who cares

Rewarding one's self or being rewarded by others for making changes
I focus my attention on one problem at a time instead of becoming overwhelmed by stress.

I keep things at home that remind me to use healthy stress management techniques.

I have someone I can count on when I experience stress in my life.

Others praise my choice to use healthy strategies to manage stress. 
Graphic presentation of data and dynamic typologies, which groups participants by their patterns of changes over time, have examined TTM constructs over time for a range of other behaviors including smoking cessation, physical activity, sun protection, and dietary fat reduction (Lipschitz et al., 2015; Norman et al.,1998; Redding et al., 2011; Sun et al.,2007; Yusufov et al., 2016a; Yusufov et al., 2016b). One TTM study compared three longitudinal patterns in a large sample of smokers, quitters, and relapsers (Sun et al., 2007). Relapsers initially performed like quitters but their use of skills then placed between quitters and smokers or non-changers (Sun et al., 2007). These patterns of change in the use of processes replicated across other health behaviors such as physical activity (Lipschitz et al., 2015), sun protection (Yusufov, 2016b), and dietary fat reduction (Yusufov et al., 2016a). Stress management change over time has not yet been examined for similar patterns.

This secondary data analysis was designed to compare TTM construct use over time for managing stress effectively across three dynatypes (Lipschitz et al., 2015; Norman et al., 1998; Redding et al., 2011; Sun et al., 2007; Yusufov et al., 2016a; Yusufov et al., 2016b). Dynatypes are defined groups with comparable longitudinal change patterns (Norman et al., 1998; Prochaska et al., 1991): those who were not managing stress effectively but then began managing stress effectively (Successful Changers); those who began managing stress effectively and then relapsed (Relapsers); and those who never changed their stress management practices (Stable Non-Changers).

This study used dynatype comparison analyses to examine TTM stress management constructs over 18 months. These analyses will examine whether patterns of TTM stress-related construct use over time were comparable to or different from those found for other health behaviors: dietary fat reduction, physical activity, smoking cessation, and sun protection. Based on previous findings from other behavioral areas, Relapsers and Maintainers were predicted to be similar at baseline on TTM variables but were expected to be doing significantly better on these variables than Stable Non-Changers. At 18 months, Relapsers' performance on all TTM processes were expected to fall in between that of Maintainers and Stable Non-Changers.

\section{Methods}

\section{Participants}

This secondary data analysis included treatment group data from a TTM-tailored stress management randomized trial (Evers et al., 2006). All participants provided informed consent and all study methods were approved by an Institutional Review Board. In the parent dataset $(n=1085)$, two directories from market research companies were purchased. Next, individuals were contacted via mail and telephone. Eligibility criteria required that individuals were over 18 years old, English-speaking, experiencing some 
degree of stress at baseline, and not currently practicing effective stress management. A recruitment rate of more than $70 \%$ was reported, including individuals from across 48 states. Additional details and outcomes are described elsewhere (Evers et al., 2006).

Participants were excluded from this study if they had completed only the baseline intervention or if they did not have all TTM constructs available for analysis. For participants who were missing some follow-up time points, the last observation (pre-Action or Action/Maintenance) from the previous time point was carried forward prior to determine their dynatype group. After data cleaning, the analytic sample included $\mathrm{N}=427$ adults who were in the treatment group and followed over time.

\section{Intervention}

The TTM-tailored intervention targeting stress management produced three computerized feedback reports at baseline, 3 and 6 months on Stage of Change for stress management and each of three TTM stress-related constructs: Decisional Balance, Self-Efficacy, and Processes of Change (Evers et al., 2006).

\section{Measures}

Demographics Gender, age, race, and marital status were assessed at baseline (Table 2).

Table 2. Baseline demographic differences between dynatype groups 


\begin{tabular}{|c|c|c|c|c|c|}
\hline & $\begin{array}{l}\text { Successful } \\
\text { Changers }\end{array}$ & Relapsers & $\begin{array}{l}\text { Stable Non- } \\
\text { Changers }\end{array}$ & & \\
\hline & $(n=252)$ & $(n=76)$ & $(n=99)$ & $x^{2}$ & $p$ \\
\hline Gender & & & & 5.72 & .057 \\
\hline Female \% & 62.6 & 67.1 & 74.9 & & \\
\hline Race & & & & 9.92 & .153 \\
\hline \multicolumn{6}{|c|}{ American Indian/ Alaskan \% } \\
\hline & 0.8 & 2.7 & 0.0 & & \\
\hline Asian/Pacific Islander \% & 0.0 & 1.4 & 0.0 & & \\
\hline Black, Non-Hispanic \% & 8.3 & 11.0 & 9.8 & & \\
\hline White, Non-Hispanic \% & 89.9 & 82.2 & 88.0 & & \\
\hline Other \% & 0.9 & 2.7 & 2.2 & & \\
\hline Hispanic & & & & 4.28 & .842 \\
\hline Yes \% & 7.7 & 1.4 & 5.1 & & \\
\hline Marital Status & & & & 5.68 & .118 \\
\hline Married \% & 64.5 & 65.8 & 66.0 & & \\
\hline $\begin{array}{l}\text { Not married, living with } \\
\text { partner } \%\end{array}$ & 3.6 & 2.6 & 4.1 & & \\
\hline Single \% & 8.5 & 9.2 & 11.3 & & \\
\hline Separated \% & 2.4 & 0.0 & 1.0 & & \\
\hline Divorced \% & 10.1 & 13.2 & 6.2 & & \\
\hline Widowed \% & 10.9 & 9.2 & 11.3 & & \\
\hline Stage of Change & & & & 16.17 & .003 \\
\hline Precontemplation & 42.1 & 65.8 & 56.6 & & \\
\hline Contemplation & 37.3 & 25.0 & 28.3 & & \\
\hline
\end{tabular}




\begin{tabular}{|c|c|c|c|c|c|}
\hline Preparation & 10.6 & 9.2 & 15.2 & & \\
\hline Age Group & & & & 13.01 & .029 \\
\hline$<34$ years $\%$ & 10.5 & 8.2 & 13.0 & & \\
\hline $35-49$ years \% & 32.9 & 32.9 & 17.4 & & \\
\hline $50-64$ years \% & 45.2 & 37.0 & 35.9 & & \\
\hline $65+$ years $\%$ & 18.9 & 21.9 & 33.7 & & \\
\hline
\end{tabular}

Note. Bold indicates $p$-value $<.05$

Stage of Change Participants were presented with the definition for effective stress management: "Stress management includes regular relaxation and physical activity, talking with others, and/or making time for social activities" and were then asked, "Do you effectively practice stress management in your daily life" (Velicer et al., 1998). Five response categories allowed participants to self-classify into one of five stages of change for stress management: Precontemplation (not intending to begin in the next 6 months), Contemplation (intending to begin in the next 6 months), Preparation (intending to begin in the next 30 days), Action (currently practicing stress management behavior, but for less than 6 months) or Maintenance (currently practicing stress management behavior for at least 6 months) (Evers et al., 2006; Prochaska et al., 2008). All eligible participants were in the pre-Action stages of change (Precontemplation, Contemplation, or Preparation) at baseline.

Decisional Balance This 12-item inventory has shown strong external validity, as well as good to excellent internal consistencies ( $a=.90$ for the Pros subscale, $a=.78$ for the Cons subscale). Participants rated the importance of each item in their decision to practice effective stress management on a five-point Likert scale ranging from $1=$ Not At All Important to $5=$ Extremely Important (Evers et al., 2006) (Table 1).

Self-Efficacy On a 10-item scale, participants rated their confidence in their ability to manage stress effectively during challenging situations (e.g., when I am feeling frustrated) on a five-point Likert scale ranging from $1=$ Not at All Confident to $5=$ Very Confident (Evers et al., 2006). Cronbach's alpha for the 10-item confidence scale was also good $(a=.874)$ (Table 1$)$.

Processes of Change The 10 processes are experiential (cognitive) or behavioral (overt) activities that individuals use to modify problem behaviors. Participants rated 30 items reflecting the 10 Processes of Change for effective stress management on a five-point Likert scale reflecting their frequency of use in the past month ( $1=$ Never; $3=$ Occasionally; $5=$ Repeatedly) (Evers et al., 2006). Overall internal consistency for Processes of Change was also good $(a=.887)$ (Table 1$)$.

\section{Dynatype Group}


Three defined groups reflecting participant change patterns over time were determined based on participants' progress from pre-Action stages at baseline to the Action or Maintenance stages at 6, 12, and 18 months post-baseline. Successful Changers included participants who were in a pre-Action stage at baseline and subsequently moved to Action or Maintenance at any follow-up time point $(6,12$, or 18 months) and remained in Action/Maintenance through 18 months. Relapsers included those who were in a pre-Action stage at baseline and moved to Action/Maintenance at one follow-up timepoint (6 or 12 months) but moved back into a pre-Action stage at 18 months. Finally, Stable Non-Changers included those who remained in pre-Action stages at all three follow-up time points $(6,12$, and 18 months).

\section{Analytic Plan}

Participants use of TTM constructs was assessed on four occasions $(0,6,12$, and 18 months) over an 18 month period and all scores were standardized to baseline scores (T-score: $M=50, S D=10$ ) to permit comparisons between constructs over time (Norman et al., 1998; Velicer, et al., 1998). Means across time for each group were plotted for each of 13 TTM constructs. Repeated measures 3 (dynatype group) X 4 (time points) MANOVAs with follow-up ANOVAs, and Tukey post-hoc tests were conducted on all TTM constructs to examine dynatype group differences. Estimates of population effect sizes were calculated using omega-squared with $90 \%$ confidence intervals (due to the nature of the $F$ distribution, which serves as the basis for calculating confidence intervals for omega-squared, a 90\% confidence interval provides $95 \%$ coverage, equivalent to an alpha level of .05) (Steiger, 2004). IBM SPSS Statistics 23 was utilized for all analyses.

\section{Results}

\section{Demographic Characteristics}

Of the total analytic sample $(n=427), 59.0 \%(n=252)$ were Successful Changers, $17.8 \%(n=76)$ were Relapsers, and $23.2 \%(n=99)$ were Stable Non-changers. No significant baseline differences were found between dynatype groups on gender, ethnicity, race, or marital status. There was a significant difference for age; Stable Non-Changers had significantly more participants over age 65 (33.7\%) compared to both Successful Changers (18.9\%) and Relapsers (21.9\%) There was also a significant difference for Stage of Change; Successful Changers included significantly fewer participants in Precontemplation $(42.1 \%)$ than both the Stable Non-Changers (56.6\%) and Relapser (65.8\%) groups (Table 2).

\section{Main Analyses Findings}

Table 3 shows MANOVA TTM construct differences with effect sizes between dynatype groups across baseline, 6, 12 and 18-months. Table 4 shows follow-up pairwise comparison results based on Tukey tests.

Table 3. Results of repeated-measure 3 (dynatype group) X 4 (time points) MANOVAs: TTM construct differences between dynatype groups at each time point 


\begin{tabular}{|c|c|c|c|c|c|}
\hline TTM Construct & & Baseline & 6 months & 12 months & 18 months \\
\hline \multirow[t]{4}{*}{ Pros } & $F(2,272)$ & 7.22 & 4.97 & 4.83 & 10.59 \\
\hline & $\omega^{2}$ & .043 & .028 & .027 & .065 \\
\hline & $90 \% \mathrm{Cl}$ & $.014, .094$ & $.006, .074$ & $.005, .072$ & $.027, .122$ \\
\hline & $p$ & .001 & .008 & .009 & $<.001$ \\
\hline \multirow[t]{4}{*}{ Cons } & $F(2,259)$ & 1.71 & 0.87 & 0.70 & 1.57 \\
\hline & $\omega^{2}$ & .005 & 0 & 0 & .004 \\
\hline & $90 \% \mathrm{Cl}$ & $0, .040$ & $0, .027$ & $0, .024$ & $0, .038$ \\
\hline & $p$ & .183 & .421 & .499 & .209 \\
\hline \multirow[t]{4}{*}{ Self-Efficacy } & $F(2,226)$ & 0.96 & 1.32 & 3.48 & 2.80 \\
\hline & $\omega^{2}$ & 0 & .003 & .021 & .016 \\
\hline & $90 \% \mathrm{Cl}$ & $0, .032$ & $0, .039$ & $.001, .070$ & $0, .061$ \\
\hline & $p$ & .385 & .268 & .032 & .063 \\
\hline \multirow[t]{4}{*}{ Consciousness Raising } & $F(2,272)$ & 11.00 & 10.01 & 27.31 & 27.51 \\
\hline & $\omega^{2}$ & .068 & .062 & .161 & .162 \\
\hline & $90 \% \mathrm{Cl}$ & $.029, .125$ & $.025, .117$ & $.102, .229$ & $.103, .231$ \\
\hline & $p$ & $<.001$ & $<.001$ & $<.001$ & $<.001$ \\
\hline \multirow[t]{4}{*}{ Dramatic Relief } & $F(2,270)$ & 3.45 & 1.59 & 10.70 & 7.07 \\
\hline & $\omega^{2}$ & .018 & .004 & .066 & .043 \\
\hline & $90 \% \mathrm{Cl}$ & $.001, .059$ & $0, .037$ & $.028, .124$ & $.013, .094$ \\
\hline & $p$ & .033 & .207 & $<.001$ & .001 \\
\hline \multirow[t]{4}{*}{ Environmental Reevaluation } & $F(2,277)$ & 9.14 & 6.92 & 20.90 & 23.44 \\
\hline & $\omega^{2}$ & .055 & .041 & .124 & .138 \\
\hline & $90 \% \mathrm{Cl}$ & $.021, .109$ & $.012, .090$ & $.072, .190$ & $.083, .205$ \\
\hline & $p$ & $<.001$ & .001 & $<.001$ & $<.001$ \\
\hline \multirow[t]{3}{*}{ Self-Reevaluation } & $F(2,270)$ & 7.29 & 10.95 & 21.42 & 26.29 \\
\hline & $\omega^{2}$ & .044 & .068 & .130 & .156 \\
\hline & $90 \% \mathrm{Cl}$ & $.014, .096$ & $.029, .126$ & $.076, .197$ & $.098, .225$ \\
\hline
\end{tabular}




\begin{tabular}{|c|c|c|c|c|c|}
\hline & $p$ & .001 & $<.001$ & $<.001$ & $<.001$ \\
\hline \multirow[t]{4}{*}{ Social Liberation } & $F(2,269)$ & 4.06 & 4.25 & 11.03 & 16.69 \\
\hline & $\omega^{2}$ & .022 & .023 & .069 & .103 \\
\hline & $90 \% \mathrm{Cl}$ & $.003, .065$ & $.003, .067$ & $.030, .127$ & $.055, .167$ \\
\hline & $p$ & .018 & .015 & $<.001$ & $<.001$ \\
\hline \multirow[t]{4}{*}{ Counter Conditioning } & $F(2,278)$ & 4.13 & 9.45 & 21.73 & 26.30 \\
\hline & $\omega^{2}$ & .022 & .057 & .129 & .153 \\
\hline & $90 \% \mathrm{Cl}$ & $.003, .064$ & $.022, .111$ & $.075, .194$ & $.095, .220$ \\
\hline & $p$ & .017 & $<.001$ & $<.001$ & $<.001$ \\
\hline \multirow[t]{4}{*}{ Stimulus Control } & $F(2,270)$ & 6.69 & 13.36 & 20.41 & 25.29 \\
\hline & $\omega^{2}$ & .040 & .083 & .125 & .151 \\
\hline & $90 \% \mathrm{Cl}$ & $.012, .090$ & $.040, .144$ & $.071, .191$ & $.093, .220$ \\
\hline & $p$ & .001 & $<.001$ & $<.001$ & $<.001$ \\
\hline \multirow[t]{4}{*}{ Helping Relationships } & $F(2,274)$ & 3.45 & 7.27 & 16.92 & 10.95 \\
\hline & $\omega^{2}$ & .017 & .043 & .103 & .067 \\
\hline & $90 \% \mathrm{Cl}$ & $.001, .058$ & $.014, .094$ & $.055, .166$ & $.029, .124$ \\
\hline & $p$ & .033 & .001 & $<.001$ & $<.001$ \\
\hline \multirow[t]{4}{*}{ Reinforcement Management } & $F(2,268)$ & 3.59 & 4.06 & 10.08 & 14.91 \\
\hline & $\omega^{2}$ & .019 & .022 & .063 & .093 \\
\hline & $90 \% \mathrm{Cl}$ & $.001, .061$ & $.003, .066$ & $.026, .120$ & $.047, .156$ \\
\hline & $p$ & .029 & .018 & $<.001$ & $<.001$ \\
\hline \multirow[t]{4}{*}{ Self-Liberation } & $F(2,274)$ & 4.69 & 10.65 & 19.63 & 31.57 \\
\hline & $\omega^{2}$ & .026 & .065 & .119 & .181 \\
\hline & $90 \% \mathrm{Cl}$ & $.005, .071$ & $.027, .122$ & $.067, .184$ & $.119, .250$ \\
\hline & $p$ & .010 & $<.001$ & $<.001$ & $<.001$ \\
\hline
\end{tabular}

Note. Bold indicates $p$-value $<.05$

Table 4. Results of follow-up ANOVAs with Tukey pairwise comparisons 


\begin{tabular}{|c|c|c|c|c|}
\hline & Baseline & 6 months & 12 months & 18 months \\
\hline Pros & $S>R, N$ & $S>R$ & $S>N$ & $S>N, R$ \\
\hline Cons & ns & ns & ns & ns \\
\hline Self-Efficacy & ns & ns & $S>R$ & ns \\
\hline $\begin{array}{l}\text { Experiential Processes } \\
\text { Consciousness Raising }\end{array}$ & $S>N, R$ & $S>N$ & $\mathrm{~S}>\mathrm{R}>\mathrm{N}$ & $S>R, N$ \\
\hline Dramatic Relief & $S>N$ & ns & $S>N$ & $S>N$ \\
\hline Environmental Reevaluation & $S>N, R$ & $S>N$ & $S>N, R$ & $S>N, R$ \\
\hline Self-Reevaluation & $S>N$ & $S>N, R$ & $S>N, R$ & $S>N, R$ \\
\hline Social Liberation & $S>N$ & $S>N$ & $S>N, R$ & $S>N, R$ \\
\hline $\begin{array}{l}\text { Behavioral Processes } \\
\text { Counter Conditioning }\end{array}$ & $S>N$ & $S>N, R$ & $\mathrm{~S}>\mathrm{R}>\mathrm{N}$ & $\mathrm{S}>\mathrm{R}>\mathrm{N}$ \\
\hline Helping Relationship & $S>N$ & $\mathrm{R}, \mathrm{S}>\mathrm{N}$ & $\mathrm{R}, \mathrm{S}>\mathrm{N}$ & $\mathrm{R}, \mathrm{S}>\mathrm{N}$ \\
\hline Reinforcement Management & ns & $\mathrm{R}, \mathrm{S}>\mathrm{N}$ & $\mathrm{R}, \mathrm{S}>\mathrm{N}$ & $S>N, R$ \\
\hline Self-Liberation & $S>N$ & $S>N, R$ & $S>N, R$ & $S>N, R$ \\
\hline Stimulus Control & $S>N, R$ & $\mathrm{R}, \mathrm{S}>\mathrm{N}$ & $S>N, R$ & $S>N, R$ \\
\hline
\end{tabular}

Note. $\mathrm{S}$ = Successful Changer; $\mathrm{R}=$ Relapser; $\mathrm{N}=$ Stable Non-Changer; $\mathrm{ns}$ = no significant differences.

\section{Decisional Balance and Self-Efficacy}

Figure 1 presents T-score patterns that emerged over 18 months for the Pros and Cons of stress management and Self-Efficacy. There were no significant differences between dynatype groups at any of the four time points on the Cons. For Pros, Relapsers showed similar stable movement to Stable NonChangers over the course of 18-months. Successful Changers had significantly greater Pros compared to Relapsers and Stable Non-Changers at one point or another and maintained the same level of Pros over 18-months. There were significant differences in Self-Efficacy across the three groups at 12-months. However, there were no significant differences in Self-Efficacy across dynatypes at baseline, 6 and 18months. At 12-months, post-hoc Tukey tests indicated that Successful Changers rated their Self-Efficacy for effective stress management as significantly greater than Relapsers, but not significantly greater than Stable Non-Changers. 
Figures 2 and 3 present mean T-scores for the five Experiential and five Behavioral Processes of Change ratings for members of each of the dynatypes, respectively. Relapsers showed more similar movement to Successful Changers than Stable Non-Changers, then typically began regressing in their process use between 6-12 months, but not as much as Stable Non-Changers. Across all Experiential Processes of Change, significant group differences were observed at all occasions, with medium to large effect sizes at most timepoints. Post-hoc Tukey tests conducted with summary scores for all Experiential processes for each dynatype group indicated that Successful Changers scored significantly higher on Experiential processes than Stable Non-changers and Relapsers at all timepoints.

Significant dynatype group differences were also observed for the Behavioral Processes at baseline, 6, 12, and 18-months, with medium to large effect sizes at most occasions. Post-hoc Tukey tests conducted with summary scores for combined Behavioral processes indicated that Successful Changers scored significantly higher on Behavioral processes than Stable Non-Changers at baseline and 6-months. At 12months, Successful Changers scored significantly higher than Relapsers and Relapsers scored significantly higher than Stable Non-Changers on combined Behavioral Processes of Change. For both the Experiential and Behavioral Processes, effect sizes tended to be larger at 12 and 18 months than at baseline and 6 months.

\section{Discussion}

All TTM stress management constructs, except the Cons, significantly differentiated between Successful Changers and Stable Non-Changers, with Relapsers falling in between these two groups. These results replicated TTM dynatype group patterns of change found for smoking cessation (Redding et al., 2011; Sun et al., 2007), sun protection (Yusufovet al., 2016b), diet (Yusufovet al., 2016a), and physical activity (Lipschitz et al., 2015), that also found Relapsers to be a distinct group. Moreover, these findings support TTM's view that experiencing a relapse can often teach individuals important skills that can help further progress towards subsequent attempts to change behavior.

More than Stable Non-Changers at all time points, Successful Changers engaged in cognitive activities such as raising awareness of the negative effects of stress or assessing how their environment was affected by not managing stress effectively. At baseline, Successful Changers used nine out of 10 cognitive and behavioral processes significantly more often than Stable Non-Changers. This supports the validity of TTM, in that using these Processes facilitated forward transitions and maintenance of stress management. In terms of Behavioral Processes of Change, Successful Changers used Counterconditioning (substitution) and Self-Liberation (choosing and commitment to act) significantly more than Non-Changers and Relapsers at all time points. Among the Processes of Change, Relapsers most consistently used Helping Relationships (seeking social support). Relapsers also increased their use of Stimulus Control (managing cues) and Counterconditioning (substitution) at 6 months, which then plateaued at 12 and 18 months. Over time, Relapsers' process use was most similar to that of Stable Non-Changers'. Stable Non-Changers' process use was significantly different than Successful Changers' process use, except for Dramatic Relief. Ultimately, Stable Non-Changers did not use behavioral 
processes as much as the other two dynatype groups and Relapsers did not sufficiently utilize cognitive and affective experiential processes.

Effect sizes for Processes of Change between dynatypes over time were large, especially at later assessments, building upon previous findings in other behavioral areas that Processes were salient predictors of stage movement (Levesque et al., 2011; Sun et al., 2007). Among Experiential Processes of Change, Consciousness Raising had medium effect sizes at baseline and 6 months, with particularly large effect sizes at 12 and 18 months. These findings indicate that attending to information related to the importance of stress management is particularly important in both the initiation and especially, maintenance of effective stress management. Effect sizes for Environmental Reevaluation and SelfReevaluation at 12 and 18 months were also large, supporting their importance during stress management maintenance. Environmental Reevaluation reflects awareness of the impact that one's own effective stress management behaviors can have on those around them. Self-Reevaluation reflects one's self image as someone who manages stress well, which these results suggest could be a powerful motivator for initiating and maintaining engagement in stress management behaviors.

Decisional Balance results were that the evaluation of the benefits of effective stress management (Pros) differentiated between dynatypes, whereas evaluation of drawbacks (Cons) did not. Pros showed a small effect size at baseline, 6 and 12 months, and a medium effect size at 18 months. At the baseline and 18month time point, Successful Changers rated Pros of stress management more highly than Stable NonChangers and Relapsers did. Over time, Successful Changers were stable in their appraisal of Pros. This finding reveals that individuals who continue to remind themselves of the benefits of stress management can sustain change over time. At 6 and 18 months, Successful Changers rated Pros as significantly more important than Relapsers. Thus, the 6 to 18 -month time period may be a critical time for Relapsers, during which intervention efforts may improve efficacy by reemphasizing the benefits of stress management. Self-Efficacy did not differentiate between dynatypes at baseline, but did show a small effect size at 6,12 and 18 months. Successful Changers' confidence improved over time and was significantly different from Relapsers at 12 months. The effect size between dynatypes from baseline to 18 months was particularly large, reflecting a threefold increase. This finding reveals that building confidence over time is especially important for both initiating and sustaining effective stress management.

Finally, there were more individuals 65 and older in the Non-Changer group compared to the other two groups. This may indicate the difficulty of changing behavior later in life or it may reflect a cohort effect in this group of older adults. More randomized research will be needed to better understand these gender and age group differences.

\section{Limitations}

This study has some limitations including sample homogeneity. Future studies should include more diverse, heterogeneous samples to replicate and extend these findings. This is particularly important given the role of culture in the perceptions of stress and coping goals, abilities and strategies (Wong, Wong, \& Lonner, 2006). 
Since these findings were based on participants from one study in one TTM-tailored treatment group, these findings may have limited generalizability. Future studies should examine other treatments longitudinally to determine whether these findings can generalize beyond this treatment. In addition, Successful Changers were the largest group in this sample (59\%), in contrast to the other two dynatype groups (Relapsers and Stable Non-Changers) which had much smaller sample sizes. Thus, the small sample sizes in the Relapsers and Stable Non-Changers groups limited statistical power to detect some differences. Finally, sociodemographic covariates such as income, education, and urban/rural status were not included here, and hence unmeasured confounders may have influenced our findings.

\section{Conclusions}

Despite these limitations, four important findings may inform future replication studies and tailored interventions for effective stress management:

1. TTM construct use facilitated stress management change, as Successful Changers used these constructs more frequently than the other groups.

2. Interventions should include reminders for participants of all the benefits of stress management uniquely for them, particularly for those who may be struggling or those with previous relapses.

3. Since Relapsers engaged in behavioral strategies more than those who did not change but did not engage in sufficient cognitive and affective strategies, interventions for individuals who have a relapse history may be more effective if they focus on cognitive strategies. Such strategies are important to utilize, given that they can cultivate intrinsic awareness and motivation to engage in and maintain behavior change.

4. Significant baseline differences between those who ultimately changed and those who did not suggest some predictions. Aside from the Cons, Self-Efficacy, and Reinforcement Management, the remaining 10 dynamic TTM process variables differentiated between Successful Changers and Stable Non-Changers at baseline (see Table 4). Future research could build upon these findings by tailoring interventions even more using these TTM constructs. This information could enable interventionists to detect Stable Non-Changers earlier, even at baseline, and then, provide enhancements that could assist them to make more progress.

In addition, based on these findings, healthcare systems and health care providers are encouraged to be more proactive and screen for or assess patients' levels of stress routinely and discuss the negative implications of chronic stress along with the range of mental health and health benefits that can be attained through effective stress management. Such an approach can also encourage timely and appropriate referrals which can both improve patient well-being and potentially reduce other healthcare costs. For one example, the National Comprehensive Cancer Network, the overseeing organization of Comprehensive Cancer Care designated hospitals, advocates routine use of a "distress thermometer" to assess current distress as part of the clinical assessment of a patient's vital signs that include weight, temperature, pulse, blood pressure, respiration, and pain. Such excellent clinical care should not be limited 
to cancer patients, but applied equally well across many settings, including primary care. Finally, encouraging the establishment of strong intrinsic rewards early in treatment is also recommended which can help an individual both adopt and maintain the practice of effective stress management longer-term.

\section{Declarations}

Ethics approval and consent to participate. All procedures followed were in accordance with the ethical standards of the responsible committee on human experimentation (institutional and national) and with the Helsinki Declaration of 1975, as revised in 2000. Informed consent for participation was obtained from all participants. All protocols were approved by the University of Rhode Island Institutional Review Board.

Consent to publication. Informed consent for publication was obtained from all participants.

Availability of data and materials. The data that support the findings of this study are available from ProChange Behaviors Systems, Inc. but restrictions apply to the availability of these data, which were used under license for the current study, and so are not publicly available. Data are however available from Kerry Evers (kevers@prochange.com) upon reasonable request and with permission of Pro-Change Behavior Systems, Inc.

Conflict of interest. Author Evers is affiliated with Pro-Change Behavior Systems, Inc. that has financial interest in the study product reported.

Authors' contributions. NSG: manuscript text; CR: conceptualization, methodology, manuscript review; KE: funding acquisition, methodology, manuscript review; JL: manuscript review; MY: manuscript review; AP: formal analysis, manuscript review; WV: conceptualization, methodology; JR: formal analysis, conceptualization, methodology, manuscript review, MR: conceptualization, manuscript review; JP: conceptualization, methodology, manuscript review.

Funding. This research was supported in part by US National Institutes of Health grant G20RR030883 from the National Center for Research Resources (PI: Donald H. DeHayes), and National Cancer Institute Small Business Innovation Research grant 1 R44CA81948-01 (PI: Kerry E. Evers).

\section{References}

1. American Psychological Association. (2017a). Stress in America: Coping with change. Stress in America $^{\text {TM }}$ Survey.

2. American Psychological Association. (2017b). Stress in America: The state of our nation. Stress in America $^{\mathrm{TM}}$ Survey.

3. American Psychological Association. (2018). Stress in America: Generation z. Stress in America ${ }^{\mathrm{TM}}$ Survey. 
4. American Psychological Association. (2019). Stress in America: Stress and current events. Stress in America $^{\text {TM }}$ Survey.

5. Baum, A., \& Posluszny, D. M. (1999). Health psychology: mapping biobehavioral contributions to health and illness. Annu Rev Psychol, 50, 137-163. doi:10.1146/annurev.psych.50.1.137

6. Blaney, C. L., Robbins, M. L., Paiva, A. L., Redding, C. A., Rossi, J. S., Blissmer, B., . . Oatley, K. (2012). Validation of the measures of the transtheoretical model for exercise in an adult African-American sample. Am J Health Promot, 26(5), 317-326. doi:10.4278/ajhp.091214-QUAN-393

7. Blissmer, B., Prochaska, J. O., Velicer, W. F., Redding, C. A., Rossi, J. S., Greene, G. W., . . Robbins, M. (2010). Common factors predicting long-term changes in multiple health behaviors. $J$ Health Psychol, 15(2), 205-214. doi:10.1177/1359105309345555

8. Evers, K. E., Prochaska, J. O., Johnson, J. L., Mauriello, L. M., Padula, J. A., \& Prochaska, J. M. (2006). A randomized clinical trial of a population- and transtheoretical model-based stress-management intervention. Health Psychol, 25(4), 521-529. doi:10.1037/0278-6133.25.4.521

9. (2019). Americans' stress, worry and anger intensified in 2018. https://news.gallup.com/poll/249098/americans-stress-worry-anger-intensified-2018.aspx

10. Hall, K. L., \& Rossi, J. S. (2008). Meta-analytic examination of the strong and weak principles across 48 health behaviors. Prev Med, 46(3), 266-274. doi:10.1016/j.ypmed.2007.11.006

11. Heber, E., Ebert, D. D., Lehr, D., Cuijpers, P., Berking, M., Nobis, S., \& Riper, H. (2017). The Benefit of Web- and Computer-Based Interventions for Stress: A Systematic Review and Meta-Analysis. $J$ Med Internet Res, 19(2), e32. doi:10.2196/jmir.5774

12. Hester, R. K., \& Miller, J. H. (2006). Computer-based tools for diagnosis and treatment of alcohol problems. Alcohol Res Health, 29(1), 36-40.

13. Johnson, S. S., Paiva, A. L., Cummins, C. O., Johnson, J. L., Dyment, S. J., Wright, J. A., . . Sherman, K. (2008). Transtheoretical model-based multiple behavior intervention for weight management: effectiveness on a population basis. Prev Med, 46(3), 238-246. doi:10.1016/j.ypmed.2007.09.010

14. Kaiser Family Foundation. (2020). KFF Health Tracking Poll - Early April 2020: The impact of coronavirus on life in America. https://www.kff.org/health-reform/report/kff-health-tracking-pollearly-april-2020/

15. Lazarus, R. S., \& Folkman, S. (1984). Stress, appraisal, and coping. New York: Springer Pub. Co.

16. Levesque, D. A., Van Marter, D. F., Schneider, R. J., Bauer, M. R., Goldberg, D. N., Prochaska, J. O., \& Prochaska, J. M. (2011). Randomized trial of a computer-tailored intervention for patients with depression. Am J Health Promot, 26(2), 77-89. doi:10.4278/ajhp.090123-QUAN-27

17. Lipschitz, J. M., Yusufov, M., Paiva, A., Redding, C. A., Rossi, J. S., Johnson, S., . . Prochaska, J. O. (2015). Transtheoretical Principles and Processes for Adopting Physical Activity: A Longitudinal 24Month Comparison of Maintainers, Relapsers, and Nonchangers. J Sport Exerc Psychol, 37(6), 592606. doi:10.1123/jsep.2014-0329 
18. Murphy, L. R. (1996). Stress management in work settings: a critical review of the health effects. Am $J$ Health Promot, 11(2), 112-135. doi:10.4278/0890-1171-11.2.112

19. Norman, G. J., Velicer, W. F., Fava, J. L., \& Prochaska, J. O. (1998). Dynamic typology clustering within the stages of change for smoking cessation. Addict Behav, 23(2), 139-153.

20. Prochaska, J. O., Butterworth, S., Redding, C. A., Burden, V., Perrin, N., Leo, M., . . Prochaska, J. M. (2008). Initial efficacy of MI, TTM tailoring and HRI's with multiple behaviors for employee health promotion. Prev Med, 46(3), 226-231. doi:10.1016/j.ypmed.2007.11.007

21. Prochaska, J. O., Velicer, W. F., Fava, J. L., Ruggiero, L., Laforge, R. G., Rossi, J. S., . . Lee, P. A. (2001). Counselor and stimulus control enhancements of a stage-matched expert system intervention for smokers in a managed care setting. Prev Med, 32(1), 23-32. doi:10.1006/pmed.2000.0767

22. Prochaska, J.O., Velicer, W.F., Guadagnoli, E., Rossi, J.S., \& DiClemente, C.C. (1991). Patterns of change: Dynamic typology applied to smoking cessation. Multivariate Behavioral Research, 26, 83107.

23. Prochaska, J. O., Velicer, W. F., Redding, C., Rossi, J. S., Goldstein, M., DePue, J., . . Plummer, B. A. (2005). Stage-based expert systems to guide a population of primary care patients to quit smoking, eat healthier, prevent skin cancer, and receive regular mammograms. Prev Med, 41(2), 406-416. doi:10.1016/j.ypmed.2004.09.050

24. Prochaska, J. O., Velicer, W. F., Rossi, J. S., Goldstein, M. G., Marcus, B. H., Rakowski, W., . . et al. (1994). Stages of change and decisional balance for 12 problem behaviors. Health Psychol, 13(1), 39-46.

25. Prochaska, J. O., Velicer, W. F., Rossi, J. S., Redding, C. A., Greene, G. W., Rossi, S. R., . . Plummer, B. A. (2004). Multiple risk expert systems interventions: impact of simultaneous stage-matched expert system interventions for smoking, high-fat diet, and sun exposure in a population of parents. Health Psychol, 23(5), 503-516. doi:10.1037/0278-6133.23.5.503

26. Redding, C. A., Prochaska, J. O., Paiva, A., Rossi, J. S., Velicer, W., Blissmer, B. J., ... Sun, X. (2011). Baseline stage, severity, and effort effects differentiate stable smokers from maintainers and relapsers. Subst Use Misuse, 46(13), 1664-1674. doi:10.3109/10826084.2011.565853

27. Richardson, K. M., \& Rothstein, H. R. (2008). Effects of occupational stress management intervention programs: a meta-analysis. J Occup Health Psychol, 13(1), 69-93. doi:10.1037/1076-8998.13.1.69

28. Steiger, J. H. (2004). Beyond the F test: Effect size confidence intervals and tests of close fit in the analysis of variance and contrast analysis. Psychol Methods, 9(2), 164-182. doi:10.1037/1082989X.9.2.164

29. Sun, X., Prochaska, J. O., Velicer, W. F., \& Laforge, R. G. (2007). Transtheoretical principles and processes for quitting smoking: a 24-month comparison of a representative sample of quitters, relapsers, and non-quitters. Addict Behav, 32(12), 2707-2726. doi:10.1016/j.addbeh.2007.04.005

30. Velicer, W. F., Diclemente, C. C., Rossi, J. S., \& Prochaska, J. O. (1990). Relapse situations and selfefficacy: an integrative model. Addict Behav, 15(3), 271-283. 
31. Velicer, W. F., Prochaska, J. O., Fava, J. L., Norman, G. L., \& Redding, C. A. (1998). Smoking cessation and stress management: Applications of the transtheoretical model of behavior change. Homeostatis, 38, 216-233.

32. Wikgren, M., Maripuu, M., Karlsson, T., Nordfjall, K., Bergdahl, J., Hultdin, J., . . Norrback, K. F. (2012). Short telomeres in depression and the general population are associated with a hypocortisolemic state. Biol Psychiatry, 71(4), 294-300. doi:10.1016/j.biopsych.2011.09.015

33. Wong, P. T. P., Wong, L. C. J., \& Lonner, W. J. (2006). Handbook of multicultural perspectives on stress and coping. New York: Springer.

34. Yusufov, M., Paiva, A. L., Redding, C. A., Lipschitz, J. M., Gokbayrak, N. S., Greene, G., . . Prochaska, J. O. (2016a). Fat Reduction Efforts: A 24-Month Longitudinal Comparison of a Large Sample of Maintainers, Relapsers, and Non-Changers. Health Promot Pract, 17(1), 116-126.

doi:10.1177/1524839915606423

35. Yusufov, M., Rossi, J. S., Redding, C. A., Yin, H. Q., Paiva, A. L., Velicer, W. F., . . Prochaska, J. 0. (2016b). Transtheoretical Model Constructs' Longitudinal Prediction of Sun Protection Over 24 Months. Int J Behav Med, 23(1), 71-83. doi:10.1007/s12529-015-9498-7

\section{Figures}



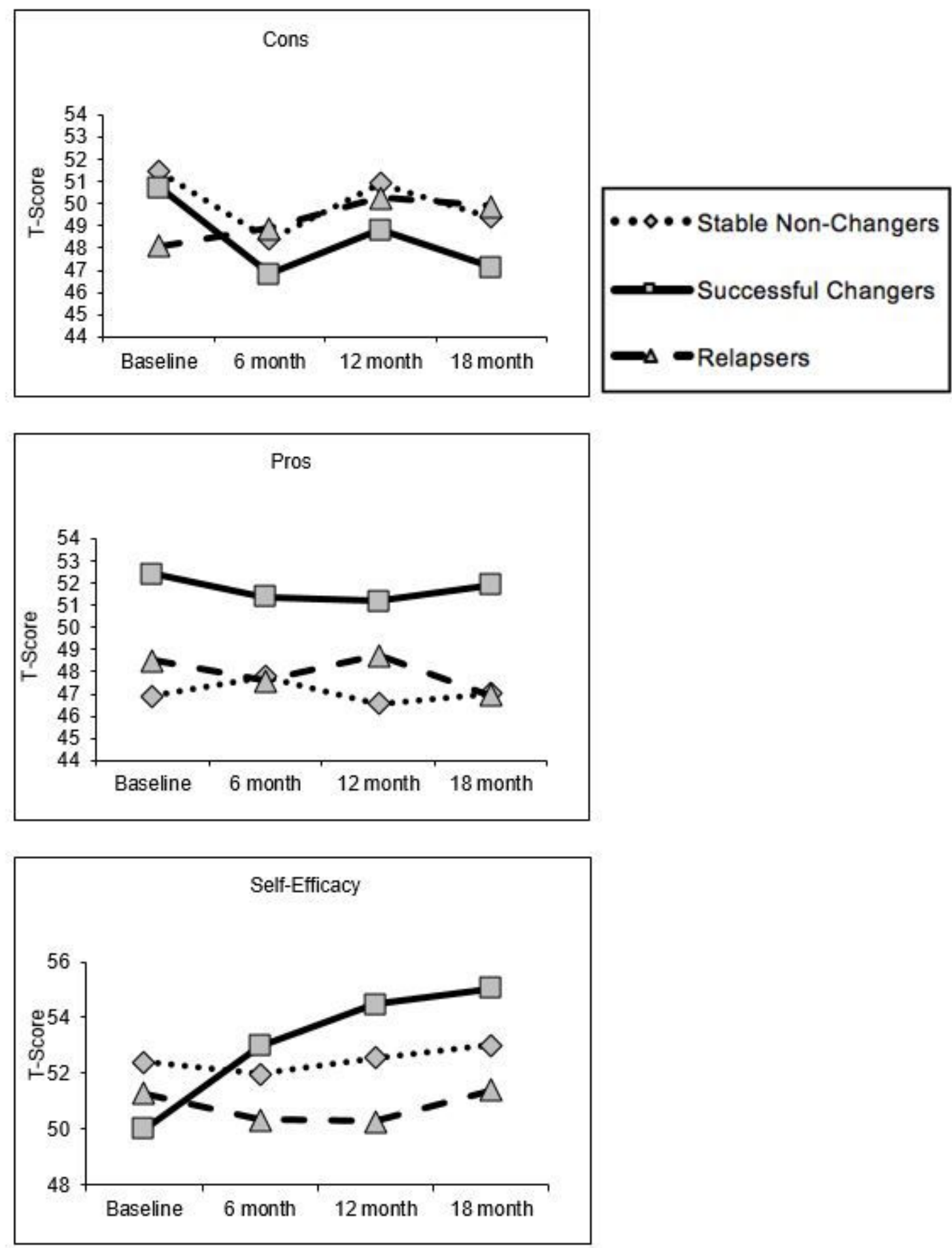

\section{Figure 1}

Decisional Balance (Pros and Cons) and Self-Efficacy construct T-scores by dynatype groups over time 

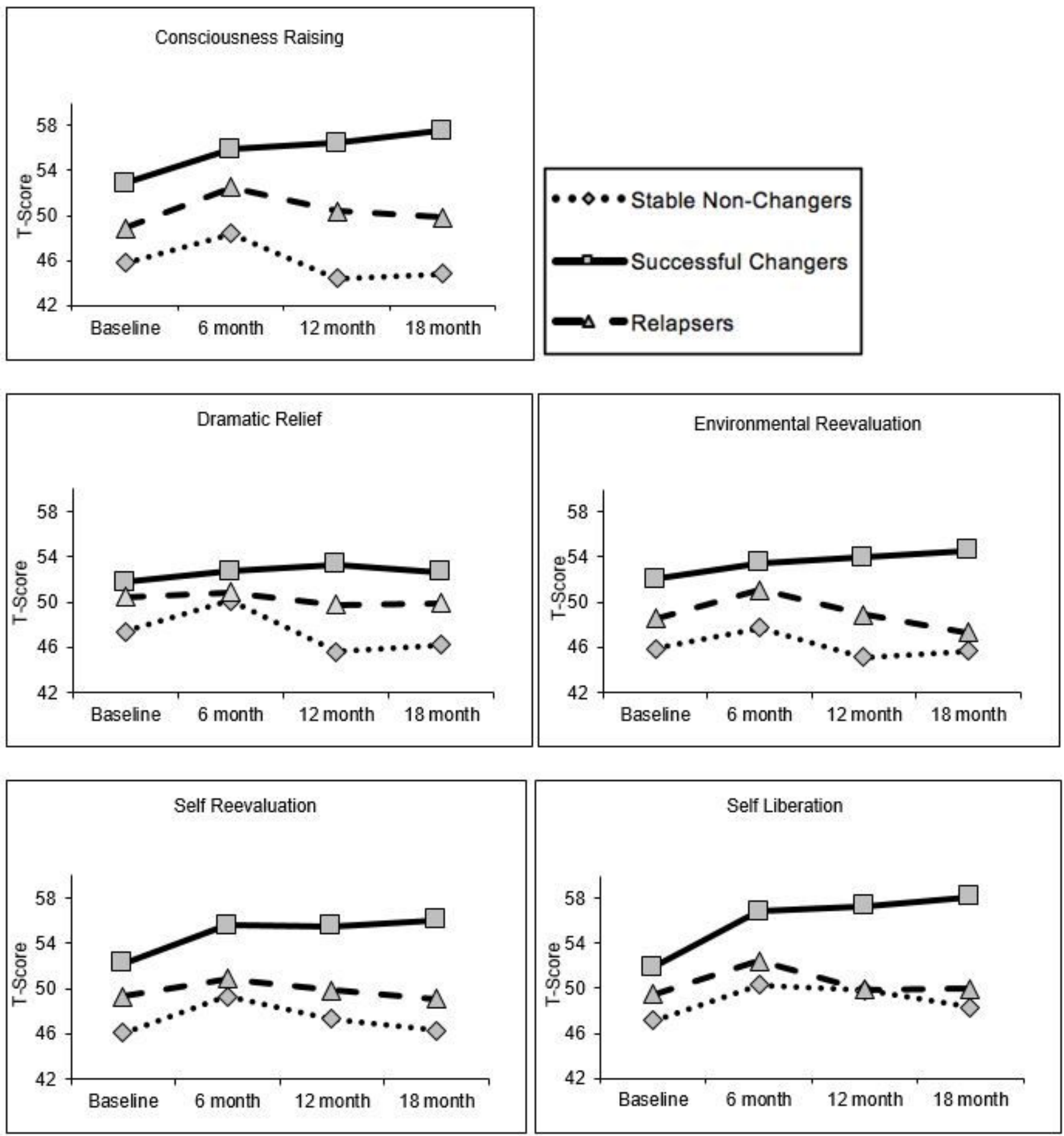

Figure 2

Experiential Processes of Change T-scores across dynatype groups over time 

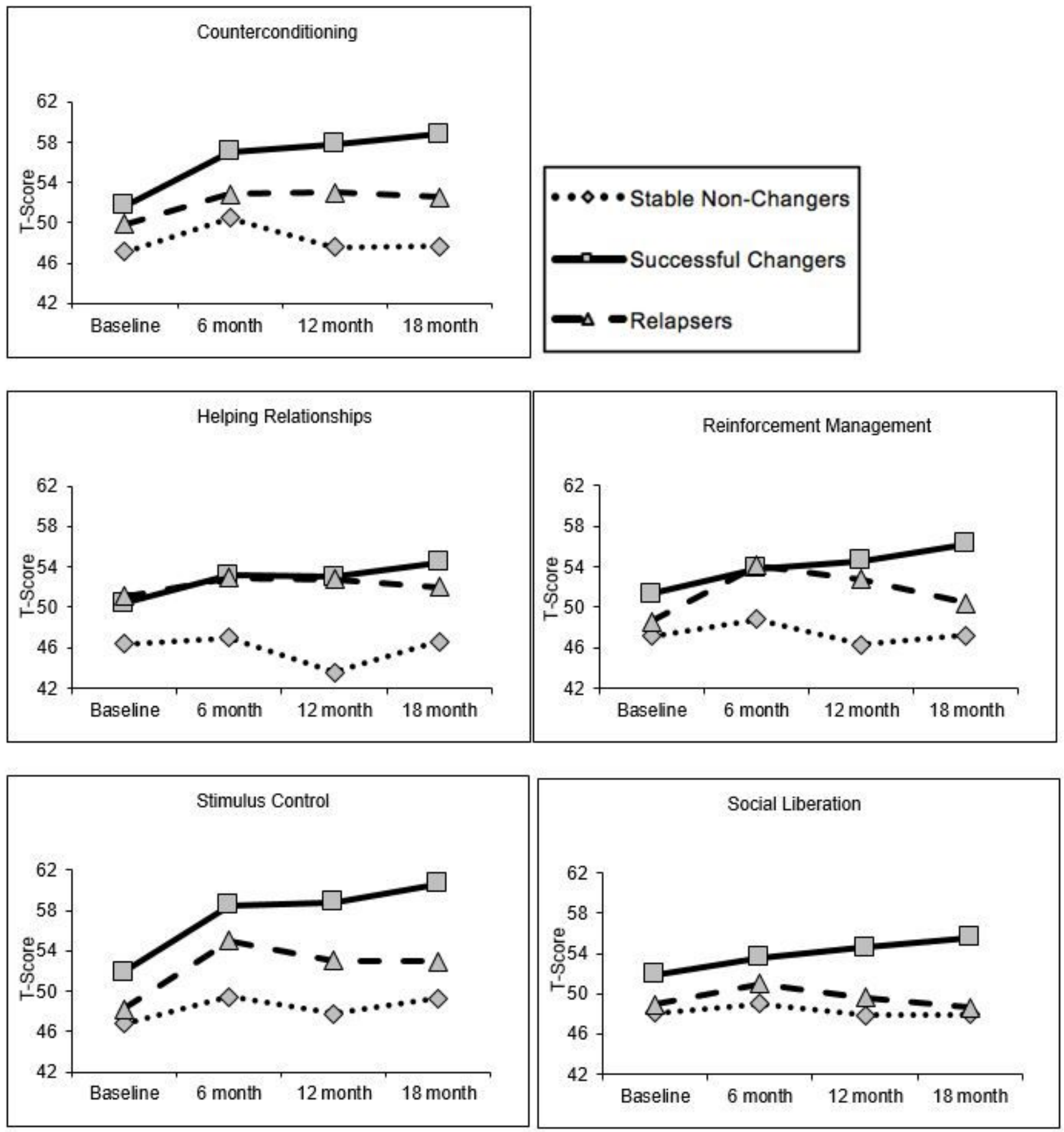

Figure 3

Behavioral Processes of Change T-scores across dynatype groups over time 